\title{
Detection of Serum miRNAs Affecting Liver Apoptosis in a Periodontitis Rat Model
}

\author{
YOSHIO SUGIURA ${ }^{1}$, TOSHIKI YONEDA ${ }^{1}$, KOHEI FUJIMORI ${ }^{1}$, TAKAYUKI MARUYAMA ${ }^{2}$, HISATAKA MIYAI $^{1}$, \\ TERUMASA KOBAYASHI ${ }^{1}$, DAISUKE EKUNI ${ }^{1}$, TAKAAKI TOMOFUJI ${ }^{3}$ and MANABU MORITA ${ }^{1}$ \\ ${ }^{1}$ Department of Preventive Dentistry, Okayama University Graduate School of Medicine, \\ Dentistry and Pharmaceutical Sciences, Okayama, Japan; \\ ${ }^{2}$ Center for Innovative Clinical Medicine, Okayama University Hospital, Okayama, Japan; \\ ${ }^{3}$ Department of Community Oral Health, Asahi University School of Dentistry, Gifu, Japan
}

\begin{abstract}
Background/Aim: miRNA molecules have been attracting attention as genetic modifiers between organs. We examined the relationship between serum miRNA and targeted liver $m R N A$ profiles in a periodontitis rat model, and the influence of periodontitis on the liver. Materials and Methods: Male Wistar rats $(n=16,8$ weeks old) were randomly divided into two groups ( 8 rats each): control and periodontitis (ligature placement for 4 weeks). Serum miRNA and liver mRNA profiles were compared. Results: Periodontal destruction and hepatocyte apoptosis were induced in the periodontitis group. Microarray analysis indicated that 52 serum miRNAs and 33 liver $m R N A s$ were expressed with a $>1.5$-fold change $(F C)$ and $a>2.0-F C(p<0.05)$, respectively, between the two groups. From the miRNA target genes, 12 genes equivalented to liver mRNAs with a $>2.0-F C$, among which, Hyoul, Chacl, and Blocls3 have apoptotic functions in our model. miRNAs upstream of these 3 mRNAs are miR3591, miR-181a-2-3p and miR-6321. Conclusion: miR-3591, miR-181a-2-3p and miR-6321 induced hepatocyte apoptosis in our periodontitis rat model.
\end{abstract}

Periodontitis is a highly prevalent inflammatory disease that occurs in tooth-supporting tissues. Hallmarks of periodontitis include i) inflammatory cell infiltration, ii) destruction of connective tissue attachment, and iii) progressive alveolar bone resorption induced by bacterial cells in biofilms located within periodontal pockets. In addition, several clinical studies

This article is freely accessible online.

Correspondence to: Toshiki Yoneda, Department of Preventive Dentistry, Okayama University Graduate School of Medicine, Dentistry and Pharmaceutical Sciences, 2-5-1 Shikata-cho, Kita-ku, Okayama 700-8558, Japan. Tel: +81 862356712, e-mail: de17057@s.okadai.jp

Key Words: MicroRNAs, periodontitis, liver. have suggested that periodontitis affects systemic diseases, such as diabetes mellitus (1), cardiovascular (2), liver (3), and kidney disease (4). Other studies have shown that periodontitis may cause liver lesions (5-8), while recently it was also reported to cause slight cell degeneration, inflammatory foci (8) and hepatocyte apoptosis in a rat periodontitis model (9). Despite all these reports, it still remains unclear what mediates the communication between periodontitis and liver pathology.

MicroRNAs (miRNAs) are endogenous noncoding regulatory RNAs comprising 17-25 nucleotides, which have critical functions in post-transcriptional gene regulation (10). miRNAs can bind complementary sequences in the 3'untranslated regions of various target messenger RNAs (mRNAs), leading to direct mRNA degradation or translational repression.

miRNAs regulate gene expression and contribute to development, differentiation, inflammation, and carcinogenesis (11). Some miRNAs have organ-specific expression (12) and stably circulate in the bloodstream (13). Therefore, circulating miRNAs from one organ can potentially change the expression of specific genes in another distant organ (14), including the liver (15); however, little is known about circulating miRNAs from periodontitis associated with pathological conditions in the liver.

We hypothesized that serum miRNAs induced by periodontitis can regulate specific mRNA expression and negatively affect the liver. The aim of this study is to examine the relationship between serum miRNA and targeted liver mRNA expression profiles, and the influence of periodontitis on the liver in a rat model.

\section{Materials and Methods}

Animals. Male Wistar rats $(\mathrm{n}=16,8$ weeks old) were housed in an air-conditioned room $\left(23-25^{\circ} \mathrm{C}\right)$ under $12-\mathrm{h} / 12$-h light-dark cycle with free access to powdered food (MF, Oriental Yeast Co. Ltd., Osaka, Japan) and drinking water. The experimental protocol was approved by the Animal Research Control Committee of Okayama 

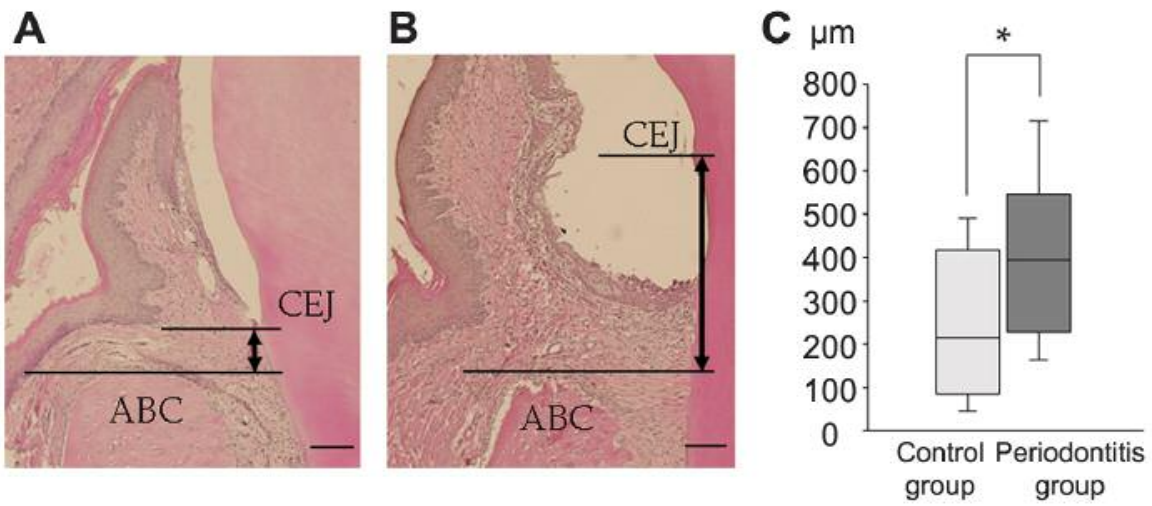

Figure 1. Progression of the alveolar bone resorption in the periodontitis group compared to the control group. Histological images of periodontal tissue by hematoxylin and eosin staining, with (A) a representative image taken of the control group at 4 weeks and (B) a representative image taken of the periodontitis group at 4 weeks. (C) Comparison of distance from CEJ to ABC between control and periodontitis groups. Box plots represent the minimum (bottom of the bar), maximum (top of the bar), median (band in the box), 25th percentile (bottom of the box), and 75th percentile (top of the box). ABC: Alveolar bone crest; CEJ: cementoenamel junction. ${ }^{*} p<0.05$, compared to the control group (Mann-Whitney U-test). Scale bar=100 $\mu$ m.

University (OKU-2014147). All animals received humane care in compliance with our institutional animal care guidelines.

Experimental design. Rats were randomly divided into a control group and a periodontitis group ( $\mathrm{n}=8$ per group). The control group received no treatment for 4 weeks. In the periodontitis group, a 5-0 silk ligature was inserted around the maxillary second molar of each animal bilaterally for 4 weeks to induce periodontitis, as previously described (8). After the experimental period, rats were euthanized and blood samples were collected from the heart. Serum was separated by centrifugation at $1,500 \times \mathrm{g}$ for $15 \mathrm{~min}$. Molar regions, including gingival tissue and liver, were resected en bloc from each rat. Molar regions were resected from the right maxilla and stored at $-80^{\circ} \mathrm{C}$ until use in histological and microarray analyses.

Histological analysis. Teeth and periodontal tissue were washed with $0.1 \mathrm{M}$ phosphate-buffered saline ( $\mathrm{pH}$ 7.4) and decalcified in OSTEOSOFT $^{\circledR}$ (EMD Millipore Corporation, Billerica, MA, USA) at $4^{\circ} \mathrm{C}$ for 3 weeks. Decalcified teeth and periodontal tissue were dehydrated in a graded alcohol series $(70 \%, 80 \%, 90 \%$, and $100 \%)$, and were then paraffin embedded. Sections $(4-\mu \mathrm{m}$ thick) in the buccolingual direction were prepared using a microtome (Yamato Kohki Industry, Saitama, Japan) and stained with hematoxylin and eosin (HE). The distance between the cementoenamel junction (CEJ) and alveolar bone crest (ABC) was then analyzed.

Resected livers were dehydrated, paraffin embedded, and sectioned as described above for teeth and periodontal tissues. Liver sections were stained with $\mathrm{HE}$ and using the terminal deoxynucleotidyl transferase-mediated dUTP nick-end labeling (TUNEL) method. To detect apoptotic cells, sections were first treated with $0.02 \mathrm{mg} / \mathrm{ml}$ proteinase K (Takara Bio Inc., Shiga, Japan) for $15 \mathrm{~min}$ at room temperature. Sections were then treated with $3 \% \mathrm{H}_{2} \mathrm{O}_{2}$ for 5 min and were incubated with a mixture of TdT enzyme and cobalt (II) chloride hexahydrate (Takara Bio Inc.) for $90 \mathrm{~min}$ at $37^{\circ} \mathrm{C}$. Then, sections were incubated with anti-fluorescein isothiocyanate horseradish peroxidase conjugate (Takara Bio Inc.) for $30 \mathrm{~min}$ at $37^{\circ} \mathrm{C}$. The color was developed with 3-30-diamino bentizine tetrahydrochloride. Finally, sections were counterstained with Mayer's hematoxylin. The number of TUNEL-positive hepatocytes as apoptotic cells was counted in 1,000 liver cells, in the area around the hepatic vein $(9,16)$.

Total RNA extraction. Total RNA, including miRNAs, was isolated from sera using the $3 \mathrm{D}-\mathrm{Gene}{ }^{\circledR}$ RNA extraction reagent from the liquid sample kit (Toray, Kanagawa, Japan) for microarray analysis. Total RNA quality was evaluated using the Agilent 2100 bioanalyzer (Agilent Technologies, Santa Clara, CA, USA). Total RNA contained $<500$ nucleotides and traces or none of $18 \mathrm{~S}$ and $28 \mathrm{~S}$ ribosomal RNA.

Liver samples were homogenized using a frozen cell crusher (Microtec Co., Chiba, Japan) and total RNA, including mRNAs, was isolated using TRIzol reagent (Invitrogen, Carlsbad, CA, USA), in accordance with the manufacturer's instruction. Total RNA quality was determined as $260 / 280 \mathrm{~mm}$ absorbance ratio of $>1.8$.

Microarray analysis. Microarray analysis using 3D-Gene (Toray) was performed in triplicates to screen candidate serum miRNAs and liver mRNAs for periodontitis. Samples from periodontitis and control groups were divided into three subgroups each: i) periodontitis $1(n=2)$, ii) periodontitis $2,(n=3)$, iii) periodontitis 3 $(n=3)$, iv) control $1(n=2), v)$ control $2(n=3)$ and vi) control $3(n=3)$. After preparing three pooled samples each, microarray analysis was conducted to investigate serum miRNA and liver mRNA profiles of each group. The numbers of mounted serum miRNAs and liver mRNAs on this microarray were 762 and 10,360, respectively. Annotation and oligonucleotide sequences of probes were based on miRBase miRNA database Release 22 (http://www.mirbase.org/). Fluorescent signals were scanned using 3D-Gene Scanner and analyzed using 3D-Gene Extraction software (Toray). Raw data for each spot were normalized by substituting with the mean intensity for background signals, which were determined from the signal intensities of blank spots. The detected spots were defined as the ones with the signal intensity over 2 standard deviations (SDs) plus mean of background signal intensity. Background-subtracted signal intensities were calculated for these spots. We used the global normalization method for background-subtracted signal intensities to set the median signal intensities at 25.0. We finally calculated FC 

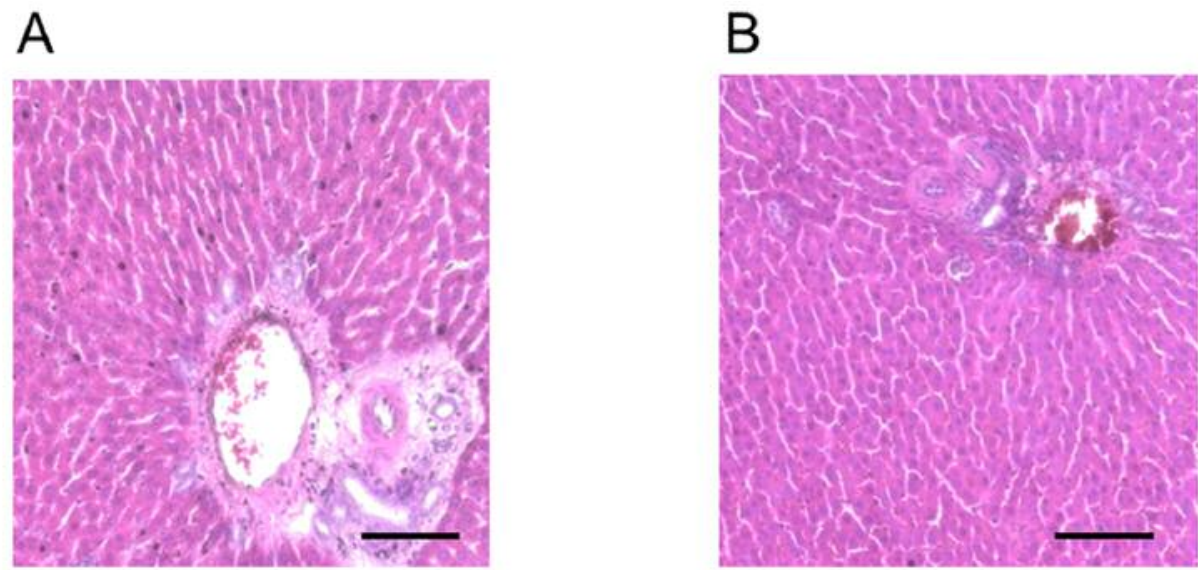

Figure 2. Representative histological images of liver stained with hematoxylin and eosin. (A) Control group and (B) Periodontitis group. No significant differences in inflammatory foci in the liver were observed between the two groups. In each histological image, a portal triad is included. Scale bar $=100 \mu \mathrm{m}$.

A

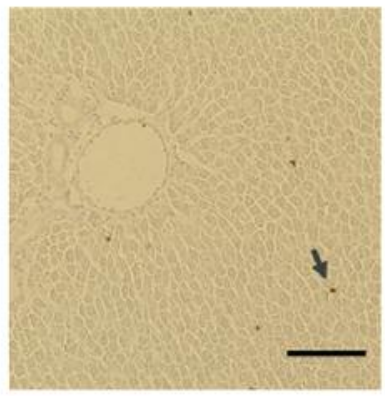

B

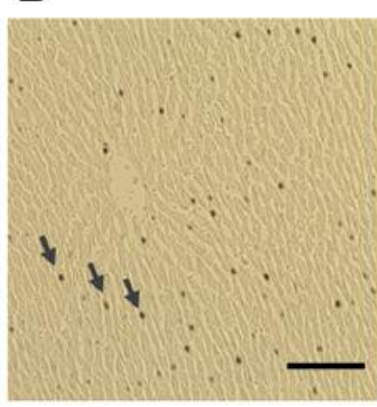

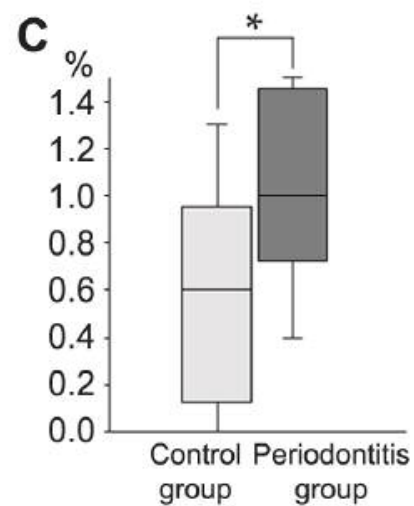

Figure 3. Increase of the TUNEL-positive hepatocytes ratio in the periodontitis group compared to the control group. Comparison of liver apoptosis between control and periodontitis groups. An increased number of TUNEL-positive hepatocytes with brown nuclei (arrowheads) was observed in the periodontitis group $(B)$ compared to the control group $(A)$. (C) Comparison of the ratio of TUNEL-positive hepatocytes in livers between control and periodontitis groups. ${ }^{*} p<0.05$, compared to the control group (Mann-Whitney U-test). Box plots represent the minimum (bottom of the bar), maximum (top of the bar), median (band in the box), 25th percentile (bottom of the box), and 75th percentile (top of the box). Scale bar=100 $\mu$ m.

values of the periodontitis group for each miRNA and mRNA using signals of the control group as reference values.

Bioinformatics analysis. Following the microarray analysis, we selected serum miRNAs with $<0.67$ or $>1.5$ FC. Next, we predicted target genes (liver mRNAs) of selected serum miRNAs using TargetScan. Among them, we further selected target genes using the following criteria: i) mRNAs $<0.5$ or $>2 \mathrm{FC}$, ii) significantly different mRNAs between periodontitis and control groups (unpaired t-test), and iii) mRNAs with apoptotic function.

Statistical analysis. Analyses were performed using the statistical package SPSS statistics 20 (IBM Japan, Tokyo, Japan). Statistical comparisons between control and periodontitis groups were carried out by the Mann-Whitney $U$-test and unpaired t-test. Differences were considered significant at $p<0.05$.

\section{Results}

Histological findings of periodontal tissues and liver. HE staining of periodontal tissues revealed that inflammatory cell penetration was slightly more severe in the periodontitis group compared to the control group at 4 weeks (Figure 1). In addition, the periodontitis group exhibited intraepithelial cleavage in the junctional epithelium (JE), whereas the control group showed no obvious pathological changes. Furthermore, the distance between the cementoenamel junction (CEJ) and alveolar bone crest $(\mathrm{ABC})$ was significantly higher in the periodontitis group compared to the control group $(p<0.05)$.

No significant differences in inflammatory foci in the liver were observed between periodontitis and control groups 
Table I. List of the differentially expressed serum miRNAs between the periodontitis and control groups.

(A) Increased expression ( $\mathrm{FC}>1.50)$

\begin{tabular}{ll}
\hline miRNAs & FC \\
\hline miR-133a-3p & 3.72 \\
miR-133b-3p & 2.95 \\
miR-206-3p & 2.75 \\
miR-493-3p & 2.45 \\
miR-344b-1-3p & 1.97 \\
miR-21-3p & 1.96 \\
miR-3591 & 1.80 \\
miR-26a-3p & 1.57 \\
miR-3084c-3p & 1.56 \\
miR-142-3p & 1.55 \\
miR-29c-5p & 1.52 \\
miR-142-5p & 1.51 \\
\hline
\end{tabular}

(B) Decreased expression $(\mathrm{FC}<0.67)$

\begin{tabular}{lc}
\hline miRNAs & FC \\
\hline let-7g-3p & 0.28 \\
miR-217-5p & 0.30 \\
miR-217-3p & 0.35 \\
miR-374-5p & 0.38 \\
miR-98-5p & 0.39 \\
miR-181a-2-3p & 0.39 \\
miR-628 & 0.40 \\
miR-146b-3p & 0.46 \\
miR-301a-5p & 0.47 \\
miR-6314 & 0.48 \\
miR-6321 & 0.48 \\
miR-1199-3p & 0.49 \\
\hline
\end{tabular}

(Figure 2). However, TUNEL staining revealed that the majority of specimens from the periodontitis group had significantly more apoptotic hepatocytes compared to those from the control group at 4 weeks $(p<0.05)$ (Figure 3$)$.

Serum miRNA profiles from periodontitis and control rats. Microarray analyses detected 762 differentially expressed miRNAs, among which 52 miRNAs were considered as aberrantly expressed with $<0.67$ or $>1.5$ FC between periodontitis and control groups at 4 weeks (Table I). In the periodontitis group, 12 miRNAs were upregulated by $>1.5$ fold compared to the control group. In addition, 40 miRNAs were downregulated in the periodontitis group compared to the control group, and these miRNAs showed $<0.67$ FC.

Liver mRNA profiles from periodontitis and control rats. Microarray analyses indicated 10,360 differentially-expressed mRNAs, among which 33 mRNAs were considered as
(B) Decreased expression $(\mathrm{FC}<0.67)$

\begin{tabular}{ll}
\hline miRNAs & FC \\
\hline miR-193a-3p & 0.50 \\
let-7a-1-3p & 0.52 \\
let-7c-2-3p & 0.52 \\
miR-23b-5p & 0.54 \\
miR-758-3p & 0.54 \\
miR-434-5p & 0.57 \\
miR-598-3p & 0.57 \\
miR-194-5p & 0.57 \\
miR-224-5p & 0.58 \\
miR-201-5p & 0.58 \\
miR-34a-3p & 0.60 \\
miR-741-3p & 0.61 \\
miR-1839-5p & 0.61 \\
miR-148a-5p & 0.62 \\
miR-192-3p & 0.62 \\
miR-421-5p & 0.62 \\
miR-362-3p & 0.62 \\
miR-216a-5p & 0.62 \\
miR-490-3p & 0.63 \\
miR-3580-5p & 0.63 \\
miR-200b-3p & 0.64 \\
miR-24-1-5p & 0.64 \\
miR-6333 & 0.64 \\
miR-183-3p & 0.65 \\
miR-28-3p & 0.65 \\
miR-98-3p & 0.65 \\
miR-6319a & 0.66 \\
\hline
\end{tabular}

FC: Fold change of miRNAs for the periodontitis group using the control group as reference.

aberrantly expressed with $<0.5$ or $>2.0 \mathrm{FC}$ and significantly different between the two groups at 4 weeks $(p<0.05$, unpaired t-test). Of these 33 mRNAs, 14 mRNAs were upregulated and 19 mRNAs were downregulated in the periodontitis group compared to the control group (Table II).

Bioinformatics Analysis. We selected liver mRNAs that are targeted by 52 serum miRNAs (Table I) using TargetScan (TargetScanHuman, Release 7.2: March 2018, http:// www.targetscan.org/vert_72/) (17). Among these target genes, 12 genes showed $<0.5$ or $>2.0 \mathrm{FC}$ ( $p<0.05$, unpaired t-test) in microarray analysis of liver mRNAs. The 12 genes were Gpr12, Hyou1, Rgma, Rad51, Dusp4, Chac1, Ier5, Ybx3, Pard6g, Bloc1s3, Sephs2, and Il7. Of these 12 genes, Hyoul, Chacl and Blocls3 exhibit an apoptotic function (18-22). miRNAs upstream of these three mRNAs are miR3591 (Hyoul), miR-181a-2-3p and miR-6321 (Chacl), miR181a-2-3p (Bloc1s3). 
Table II. List of the differentially expressed liver mRNAs between the periodontitis and control groups.

(A) Increased expression ( $\mathrm{FC}>2.0)$

\begin{tabular}{lll}
\hline mRNAs symbol & Description & FC \\
\hline Dusp4 & dual specificity phosphatase 4 & 2.94 \\
Ier5 & immediate early response 5 & 2.9 \\
Sephs 2 & selenophosphate synthetase 2 & 2.83 \\
Hbq1b & hemoglobin, theta 1B & 2.35 \\
Ahsp & alpha hemoglobin stabilizing protein & 2.35 \\
Chac1 & ChaC glutathione-specific gamma-glutamylcyclotransferase 1 & 2.31 \\
Il7 & interleukin 7 & 2.31 \\
Sigirr & single immunoglobulin and toll-interleukin 1 receptor (TIR) domain & 2.23 \\
Bloc1s3 & biogenesis of lysosomal organelles complex-1, subunit 3 & 2.18 \\
Zfp536 & zinc finger protein 536 & 2.08 \\
Zfpm1 & zinc finger protein, multitype 1 & 2.08 \\
Ybx3 & Y box binding protein 3 & 2.07 \\
Pard6g & par-6 family cell polarity regulator gamma & 2.05 \\
LOC500567 & similar to RIKEN cDNA 1700029M20 & 2 \\
\hline
\end{tabular}

(B) Decreased expression $(\mathrm{FC}<0.5)$

\begin{tabular}{|c|c|c|}
\hline mRNAs symbol & Description & $\mathrm{FC}$ \\
\hline Olr515 & olfactory receptor 515 & 0.31 \\
\hline$H s d 17 b 14$ & hydroxysteroid (17-beta) dehydrogenase 14 & 0.38 \\
\hline Gpr12 & $G$ protein-coupled receptor 12 & 0.41 \\
\hline$R G D 1561161$ & similar to BC067074 protein & 0.42 \\
\hline $\operatorname{Rad51}$ & RAD51 recombinase & 0.43 \\
\hline Fancg & Fanconi anemia, complementation group $G$ & 0.43 \\
\hline Klri2 & killer cell lectin-like receptor family I member 2 & 0.46 \\
\hline Pdia5 & protein disulfide isomerase family A, member 5 & 0.46 \\
\hline Ifitm5 & interferon induced transmembrane protein 5 & 0.46 \\
\hline Slcla6 & solute carrier family 1 member 6 & 0.47 \\
\hline Klf3 & Kruppel-like factor 3 (basic) & 0.47 \\
\hline Ascl3 & achaete-scute family bHLH transcription factor 3 & 0.49 \\
\hline Olr441 & olfactory receptor 441 & 0.49 \\
\hline Rgma & repulsive guidance molecule family member $A$ & 0.49 \\
\hline $\operatorname{Prpf8}$ & pre-mRNA processing factor 8 & 0.49 \\
\hline$R G D 1561552$ & similar to WASP (Wiskott-Aldrich Syndrome protein) family 1 & 0.49 \\
\hline Kcnabl & potassium voltage-gated channel subfamily A member regulatory beta subunit 1 & 0.49 \\
\hline$T s n$ & Translin & 0.49 \\
\hline Hyou1 & hypoxia up-regulated 1 & 0.50 \\
\hline
\end{tabular}

FC: Fold change of mRNAs for the periodontitis group using the control group as reference.

\section{Discussion}

It has been shown that miRNAs derived from some organs can affect circulating miRNAs and subsequently change mRNA expression in other distant organs, such as adipose tissue generating specific miRNAs that circulate in the bloodstream (14). Through these circulating miRNAs, adipose tissue reduces the mRNA of Fibroblast Growth Factor 21 (FGF21) in the liver, muscles, and pancreas, leading to metabolic changes, such as increased glucose tolerance (14). In the present study, we investigated the relationship between serum miRNA and targeted liver mRNA profiles, and the influence of periodontitis on liver in a rat model. This study showed that serum miRNA (miR-3591, miR-181a-2-3p, and miR-6321) and liver mRNA (Hyoul, Chacl, and Blocls3) profiles changed in periodontitis, and hepatocyte apoptosis was induced. To our knowledge, this is the first study to show the possibility of serum miRNA and liver mRNA involvement in periodontitis-induced hepatocyte apoptosis.

We found that the percentage of TUNEL-positive hepatocytes was significantly higher in the periodontitis group compared to the control group, which confirms the findings of another study that has used a rat periodontitis model (9). 
We performed a bioinformatics analysis and found that serum miRNAs specifically targeted 12 liver mRNAs: Gpr12, Hyou1, Rgma, Rad51, Dusp4, Chac1, Ier5, Ybx3, Pard6g, Bloc1s3, Sephs2, and Il7. Of these liver mRNAs, Hyoul, Chacl and Blocls3 have apoptotic function in hepatocytes. miR-3591 targets Hyoul, miR-181a-2-3p targets Chacl and Blocls3, and miR-6321 targets Chac1. These results indicate that periodontitis could change the profile of serum miRNAs and specifically regulate liver mRNAs involved in hepatocyte apoptosis.

Hyoul is involved in protein folding in the endoplasmic reticulum (ER) and suppresses ER stress (18). Chacl plays a role in inducing cells under high ER stress to undergo apoptosis (19). ER stress is caused by the accumulation of misfolded proteins in the ER. Under excess stress, cells are induced to undergo apoptosis (20). In this study, Hyoul expression (suppresses ER stress) was downregulated, while Chacl expression (induces apoptosis in cells under high ER stress) was upregulated. Therefore, we speculate that Hyoul and Chacl induce hepatocyte apoptosis through ER stress regulation.

Blocls3 can activate natural killer (NK) cells (21), and NK cells can worsen non-alcoholic liver disease (NAFLD) (23). In this study, Bloc1s3 expression was upregulated. However, liver inflammation was not clear in HE staining as mentioned above and NAFLD did not occur. Therefore, we considered that Blocls3 did not actually lead to pathological inflammatory changes. On the other hand, it is reported that NK cells induce tumor cell apoptosis (22). Considering NK cells are immune cells, Bloc 1 s 3 could have an apoptotic function for a non-selfcell, such as in tumor cells, through the activation of NK cells as part of the immune response. Therefore, Bloc $1 s 3$ may be related to apoptosis even in non-neoplastic liver cells as well as in slightly denatured periodontitis-induced liver cells.

One other reason why inflammation of the liver was not observed in our model, is that thinner sutures induced milder periodontitis, which is thought to cause little liver inflammation. In our study, a 5-0 silk ligature was inserted around the maxillary second molar of each animal instead of a 3-0 ligature used in previous studies $(8,24)$, which identified inflammation.

Specific serum miRNAs (miR-207, miR-376b-3p, and miR-495) have been previously identified as valuable periodontitis biomarkers by investigating the change in serum miRNA profile in the periodontitis rat model (8). However, these miRNAs were different from miRNAs identified in our study (miR-3591, miR-181a-2-3p, and miR-6321). A possible reason for this may still be the degree of inflammation, however, this needs to be validated in future studies.

This study has some limitations. First, we did not examine miR-3591, miR-181a-2-3p, and miR-6321 expression levels in periodontal tissues, thus, it is still unclear whether changes in serum miRNA profile actually reflect those in tissues. Second, we only performed bioinformatics analysis to determine the relationship between serum miRNAs and liver mRNAs. Detailed roles of these miRNAs should be investigated in future studies.

In conclusion, hepatocyte apoptosis was induced by periodontitis. We found that serum miRNA and liver mRNA profiles were altered in periodontitis rats compared to the control rats. Among these, serum miRNAs: i) miR-3591, ii) miR-181a-2-3p and iii) miR-6321 target "Hyoul," "Chacl and Bloc1s3," and "Chac1," respectively. These mRNAs are involved in the induction of apoptosis in hepatocytes.

\section{Conflicts of Interest}

The Authors have no conflicts of interest to declare.

\section{Authors' Contributions}

YS, TY, DE, TT, and MM conceived and designed the experiments, YS and HM performed the experiments, YS, KF, TM, and TK analyzed the data; YS, TY, DE, and MM wrote the paper.

\section{Acknowledgements}

This work was supported by a Grant-in-Aid for Scientific Research (16K11855) from the Japan Society for the Promotion of Science, Tokyo, Japan.

\section{References}

1 Sgolastra F, Severino M, Pietropaoli D, Gatto R and Monaco A: Effectiveness of periodontal treatment to improve metabolic control in patients with chronic periodontitis and type 2 diabetes: a meta-analysis of randomized clinical trials. J Periodontol 84 : 958-973, 2013. PMID: 23106512. DOI: 10.1902/jop.2012.120377

2 Ramırez J H, Arce RM and Contreras A: Periodontal treatment effects on endothelial function and cardiovascular disease biomarkers in subjects with chronic periodontitis: protocol for a randomized clinical trial. Trials 12: 46, 2011. PMID: 21324167. DOI: $10.1186 / 1745-6215-12-46$

3 Nagao Y, Kawahigashi Y and Sata M: Association of periodontal diseases and liver fibrosis in patients with $\mathrm{HCV}$ and/or HBV infection. Hepat Mon 14: e23264, 2014. PMID: 25737729. DOI: 10.5812/hepatmon.23264

4 Ismail G, Dumitriu HT, Dumitriu AS and Ismail FB: Periodontal disease: a covert source of inflammation in chronic kidney disease patients. Int J Nephrol 2013: 515796. PMID: 23840952. DOI: $10.1155 / 2013 / 515796$

5 Mester A, Ciobanu L, Taulescu M, Apostu D, Lucaciu O, Filip GA, Feldrihan V, Licarete E, Ilea A, Piciu A, Oltean-Dan D, Scurtu I, Berce C and Campian RS: Periodontal disease may induce liver fibrosis in an experimental study on Wistar rats. J Periodontol 90(8): 911-919, 2019. PMID: 30689209. DOI: 10.1002/JPER.18-0585

6 Dos Santos Carvalho J, Cardoso Guimarães Vasconcelos AC, Herlany Pereira Alves E, Dos Santos Carvalho A, da Silva FRP, de Carvalho França LF, de Pádua Rocha Nóbrega Neto A, Di Lenardo D, de Souza LKM, Barbosa ALDR, Medeiros JR, de 
Oliveira JS and Vasconcelos DFP: Steatosis caused by experimental periodontitis is reversible after removal of ligature in rats. J Periodontal Res 52(5): 883-892, 2017. PMID: 28504459. DOI: $10.1111 /$ jre. 12459

7 Tomofuji T, Sanbe T, Ekuni D, Azuma T, Irie K, Maruyama T, Tamaki $\mathrm{N}$ and Yamamoto $\mathrm{T}$ : Oxidative damage of rat liver induced by ligature-induced periodontitis and chronic ethanol consumption. Arch Oral Biol 53(12): 1113-1118, 2008. PMID: 18603227. DOI: 10.1016/j.archoralbio.2008.05.015

8 Tomofuji T, Yoneda T, Machida T, Ekuni D, Azuma T, Kataoka $\mathrm{K}$, Maruyama $\mathrm{T}$ and Morita $\mathrm{M}$ : MicroRNAs as serum biomarkers for periodontitis. J Clin Periodontol 43: 418-425, 2016. PMID: 26910654. DOI: 10.1111/jcpe. 12536

9 Tomofuji T, Ekuni D, Yamanaka R, Kusano H, Azuma T, Sanbe T, Tamaki N, Yamamoto T, Watanabe T, Miyauchi M and Takata $\mathrm{T}$ : Chronic administration of lipopolysaccharide and proteases induces periodontal inflammation and hepatic steatosis in rats. $\mathrm{J}$ Periodontol 78(10): 1999-2006, 2007. PMID: 17916001. DOI: 10.1902/jop.2007.070056

10 Stark A, Bushati N, Jan $\mathrm{CH}$, Kheradpour P, Hodges E, Brennecke J, Bartel DP, Cohen SM and Kellis M: A single Hox locus in Drosophila produces functional microRNAs from opposite DNA strands. Genes Dev 22: 8-13, 2008. PMID: 18172160. DOI: $10.1101 / \mathrm{gad} .1613108$

11 Raisch J, Darfeuille-Michaud A and Thu Nguyen HT: Role of microRNAs in the immune system, inflammation and cancer. World J Gastroenterol 19(20): 2985-2996, 2013. PMID: 23716978. DOI: $10.3748 / w j g . v 19 . i 20.2985$

12 Zhou M, Hara H, Dai Y, Mou L, Cooper DK, Wu C and Cai Z: Circulating organ-specific microRNAs serve as biomarkers in organ-specific diseases: Implications for organ allo- and xenotransplantation. Int J Mol Sci 17(8): 2016. E1232. PMID: 27490531. DOI: $10.3390 /$ ijms17081232

13 Arroyo JD, Chevillet JR, Kroh EM, Ruf I, Pritchard CC, Gibson DF, Mitchell PS, Bennett CF, Pogosova-Agadjanyan EL, Stirewalt DL, Tait JF and Tewari M: Argonaute 2 complexes carry a population of circulating microRNAs independent of vesicles in human plasma. Proc Natl Acad Sci U S A 108: 50035008, 2011. PMID: 21383194. DOI: 10.1073/pnas. 1019055108

14 Thomou T, Mori MA, Dreyfuss JM, Konishi M, Sakaguchi M, Wolfrum C, Rao TN, Winnay JN, Garcia-Martin R, Grinspoon SK, Gorden P and Kahn CR: Corrigendum: Adipose-derived circulating miRNAs regulate gene expression in other tissues. Nature 545(7653): 252, 2017. PMID: 28492253. DOI: 10.1038/ nature 22319

15 Miao C, Xie Z and Chang J: Critical roles of microRNAs in the pathogenesis of fatty liver: New advances, challenges, and potential directions. Biochem Genet 56(5): 423-449, 2018. PMID: 29951838. DOI: 10.1007/s10528-018-9870-9
16 Ekuni D, Endo Y, Irie K, Azuma T, Tamaki N, Tomofuji T and Morita M: Imbalance of oxidative/anti-oxidative status induced by periodontitis is involved in apoptosis of rat submandibular glands. Arch Oral Biol 55(2): 170-176, 2010. PMID: 20035925. DOI: 10.1016/j.archoralbio.2009.11.013

17 Agarwal V, Bell G W, Nam JW and Bartel DP: Predicting effective microRNA target sites in mammalian mRNAs. Elife 4: 2015. PMID: 26267216. DOI: 10.7554/eLife.05005

18 Kitao Y, Hashimoto K, Matsuyama T, Iso H, Tamatani T, Hori O, Stern DM, Kano M, Ozawa K and Ogawa S: ORP150/ HSP12A regulates Purkinje cell survival: a role for endoplasmic reticulum stress in cerebellar development. J Neurosci 24(6): 14861496, 2004. PMID: 14960622. DOI: 10.1523/JNEUROSCI.402903.2004

19 Mungrue IN, Pagnon J, Kohannim O, Gargalovic PS and Lusis AJ: CHAC1/MGC4504 is a novel proapoptotic component of the unfolded protein response, downstream of the ATF4-ATF3CHOP cascade. J Immunol 182(1): 466-476, 2009. PMID: 19109178. DOI: 10.4049/jimmunol.182.1.466

20 Tabas I and Ron D: Integrating the mechanisms of apoptosis induced by endoplasmic reticulum stress. Nat Cell Biol 13: 184190, 2011. PMID: 21364565. DOI: $10.1038 /$ ncb0311-184

21 Orn A, Håkansson EM, Gidlund M, Ramstedt U, Axberg I, Wigzell $\mathrm{H}$ and Lundin LG: Pigment mutations in the mouse which also affect lysosomal functions lead to suppressed natural killer cell activity. Scand J Immunol 15(3): 305-310, 1982. PMID: 7089489. DOI: 10.1111/j.1365-3083.1982.tb00653.x

22 Chollat-Namy M, Ben Safta-Saadoun T, Haferssas D, Meurice G, Chouaib S and Thiery J: The pharmalogical reactivation of p53 function improves breast tumor cell lysis by granzyme B and NK cells through induction of autophagy. Cell Death Dis 10(10): 695, 2019. PMID: 31541080. DOI: 10.1038/s41419-0191950-1

23 Tajiri K and Shimizu Y: Role of NKT Cells in the Pathogenesis of NAFLD. Int J Hepatol 2012. PMID: 22577564. DOI: $10.1155 / 2012 / 850836$

24 Tomofuji T, Ekuni D, Sanbe T, Irie K, Azuma T, Maruyama T, Tamaki N, Murakami J, Kokeguchi S and Yamamoto T: Effects of vitamin $\mathrm{C}$ intake on gingival oxidative stress in rat periodontitis. Free Radic Biol Med 46(2): 163-168, 2009. PMID: 18983910. DOI: $10.1016 /$ j.freeradbiomed.2008.09.040

Received October 19, 2019

Revised November 5, 2019

Accepted November 6, 2019 